\title{
Testing the Potential of Regulatory Sigma Factor Mutants for Wastewater Purification or Bioreactor Run in High Light
}

\author{
Dimitar Valev ${ }^{1}$ Juha Kurkela ${ }^{1}$ Esa Tyystjärvi ${ }^{1} \cdot$ Taina Tyystjärvi $^{1}$
}

Received: 4 August 2019 / Accepted: 27 March 2020 / Published online: 7 April 2020

(c) The Author(s) 2020

\begin{abstract}
It is shown that a freshly inoculated culture of the model cyanobacterium Synechocystis sp. PCC 6803 consumed almost all phosphate and $50 \%$ of nitrate within 6 days from the nutrient-rich BG-11 growth medium, indicating potential of cyanobacteria to purify wastewaters. Synechocystis sp. PCC 6803 control strain also collected nutrients efficiently from a landfill leachate wastewater KA2 (5.9-6.9 mM ammonium and 0.073-0.077 mM phosphate). Wastewaters might induce oxidative stress to microalgae, which prompted us to test growth of sigma factor inactivation strains, as $\Delta$ sigBCE and $\Delta$ sigCDE strains show superior growth in chemically induced oxidative stress. All cyanobacterial strains, including a stress-sensitive strain $\Delta$ sigBCDE, grew well in KA2 for four days, indicating that KA2 did not cause immediate oxidative stress. Completely arrested growth and bleaching of $\triangle$ sigBCDE cells after one week in KA2 wastewater point to the importance of group 2 sigma factor-mediated changes in gene expression during wastewater treatment. The growth of $\triangle$ sigBCD was arrested early in un-buffered and Hepes buffered (pH 7.5) KA2. In $\triangle$ sigBCD, all phosphate transporter genes are upregulated in standard conditions, and $\triangle$ sigBCD cells showed growth defects in low-phosphate BG-11 medium. $\Delta$ sigBCD cells removed phosphate slower from KA2 than the control strain, but phosphate supplementation of KA2 did not improve growth of $\Delta \operatorname{sigBCD}$. The $\triangle$ sigBCE strain showed superior growth in a laboratory-scale bioreactor in bright light and removed phosphate even slightly more efficiently than the control strain if KA2 was Hepes buffered although $\triangle$ sigBCE grew slowly in un-buffered KA2 and in low-phosphate BG-11 medium. The results indicate that engineering expression of regulatory group 2 sigma factor(s) might be useful for practical applications.
\end{abstract}

\section{Introduction}

Concerns about the environment have promoted research aiming at finding alternatives for efficient recycling of resources and production of food and fuel in a carbon neutral way. Municipal, piggery and dairy wastewaters, and anaerobic digestion reject waters are just few examples of nutrientrich wastewaters, containing plenty of ammonium, nitrate, and phosphate $[1,2]$. Such wastewaters cause eutrophication of natural waters. Microalgal-based wastewater treatment might offer an alternative or supplement to the conventional

Electronic supplementary material The online version of this article (https://doi.org/10.1007/s00284-020-01973-w) contains supplementary material, which is available to authorized users.

Taina Tyystjärvi

taityy@utu.fi

1 Department of Biochemistry/Molecular Plant Biology, University of Turku, 20014 Turku, Finland wastewater treatment processes. In the simplest approach for wastewater purification with microalgae, the algae absorb inorganic nutrients and utilize those for biomass production, for a review see [3]. Biomass, in turn, can be used for energy production or as a fertilizer. In a more ambitious scenario, cyanobacteria could be engineered to produce high-value compounds, not only biomass, and simultaneously remediate wastewaters.

Cyanobacteria can utilize nitrate [4], ammonium [5], and urea [6] as their nitrogen source. Ammonium ion enters the Synechocystis sp. PCC 6803 cell via permeases Amt1, 2, and 3 [7]. The nitrite--nitrate transporter NTR takes in nitrate and then nitrate reductase NarB reduces it to nitrite and nitrite reductase NirA further on to ammonium, for a recent review see [8]. Although cyanobacteria prefer ammonium, high concentrations of ammonium are toxic. Synechocystis sp. PCC 6803 grows similarly in high $\mathrm{CO}_{2}$ whether $17.6 \mathrm{mM}$ ammonium or $17.6 \mathrm{mM} \mathrm{NaNO}_{3}$ is used as a nitrogen source [9], but in ambient air, ammonium concentrations above $10 \mathrm{mM}$ adversely affect PSII activity [10]. If excess nitrogen 
is available, then cyanobacteria balance metabolism by producing the storage compound cyanophycin [11].

The other main nutrient, phosphorus, is taken up by two phosphate-specific transporters, Pst1 and Pst2 [12]. The main transporter, Pst1, is induced during phosphate limitation [13]. When excess of inorganic phosphate is available, cyanobacteria store phosphate as polyphosphate and then utilize it during phosphate starvation using two inducible enzymes exopolyphosphatase Ppx and inorganic pyrophosphorylase Ppa [14]. Phosphate metabolism is regulated by a two-component system consisting of a histidine kinase $\mathrm{SphS}$ and a response regulator $\mathrm{SphR}$ and by a negative regulator SphU [15-17]. To achieve fast and stable growth, aquatic phototrophs require adequate concentrations of $\mathrm{N}$ and $\mathrm{P}$ and in some cases $\mathrm{P}$ or $\mathrm{N}$ have to be adjusted for long-term cultivations in wastewaters $[18,19]$.

For biotechnical applications, robust strains with high acclimation capacity to suboptimal conditions are beneficial, as keeping up constant optimal environmental conditions is expensive. Acclimation of cyanobacteria to suboptimal conditions depends strongly on changes in gene expression. In cyanobacteria, the regulatory $\sigma$ subunits of the RNA polymerase (RNAP) play major roles in acclimation responses [20]. In optimal growth conditions, the RNAP core mainly recruits the primary $\sigma$ factor that guides RNAP to transcribe housekeeping genes, while in suboptimal conditions, alternative $\sigma$ factors are recruited more regularly, and the expression of genes necessary for acclimation to that particular stress is upregulated [21]. Biotechnical engineering of $\sigma$ factors might be useful to produce more robust expression strains and offer a way to control production of valuable compounds [22]

The model cyanobacterium Synechocystis sp. PCC contains nine $\sigma$ factors. SigA is an essential primary $\sigma$ factor; $\operatorname{SigB}$, $\mathrm{SigC}, \mathrm{SigD}$, and SigE are group $2 \sigma$ factors; and SigF, SigG, $\mathrm{SigH}$, and SigI are group $3 \sigma$ factors [20]. Recent studies indicate importance of group $2 \sigma$ factors in acclimation processes. Synechocystis s. PCC 6803 cells grow well without group $2 \sigma$ factors in optimal conditions, but the group $2 \sigma$ factor-deficient $\Delta$ sigBCDE cells have lost their capacity to acclimate to stress conditions [21] (see Table 1). The current knowledge about the phenotypes of the triple inactivation strains containing only one functional group $2 \sigma$ factor have been collected to Table 1 . The SigB factor plays a crucial role when cells acclimate to heat $[23,24]$ or high salt $[25,26]$ stresses. Furthermore, a high SigB content protects cells against photoinhibition $[27,28]$ and toxicity of butanol [29] and offers protection against hydrogen peroxide stress and high temperature $[29,30]$. Accumulation
Table 1 Properties of group 2 $\sigma$ factor triple and quadruple inactivation strains of Synechocystis sp. PCC 6803

\begin{tabular}{|c|c|c|c|}
\hline Strain & Group $2 \sigma$ factor content & Phenotype & References \\
\hline \multirow[t]{5}{*}{$\Delta \operatorname{sigBCD}$} & \multirow[t]{5}{*}{ SigE, $1.5 \times$ that in $\mathrm{CS}$} & Sensitive to superoxide & {$[30]$} \\
\hline & & Salt sensitive & [26] \\
\hline & & Low temperature sensitive & {$[37]$} \\
\hline & & Sensitive to PSII damage & {$[37]$} \\
\hline & & Slow growth in mixotrophic conditions & [37] \\
\hline \multirow[t]{6}{*}{$\Delta$ sigBCE } & \multirow[t]{6}{*}{ SigD, $2 \times$ that in $C S$} & Resistant to singlet oxygen stress & {$[30]$} \\
\hline & & Resistant to $\mathrm{H}_{2} \mathrm{O}_{2}$ stress & {$[30]$} \\
\hline & & Fast growth in moderate and bright light & {$[26,30]$} \\
\hline & & Sensitive to PSII damage & {$[37]$} \\
\hline & & Slow growth in blue light & {$[37]$} \\
\hline & & Locked in state 1 & [37] \\
\hline \multirow[t]{5}{*}{$\Delta \operatorname{sigBDE}$} & \multirow[t]{5}{*}{ Normal amount of SigC } & Sensitive to superoxide & {$[30]$} \\
\hline & & Low temperature sensitive & {$[37]$} \\
\hline & & Sensitive to PSII damage & {$[37]$} \\
\hline & & Slow recovery from nitrogen deficiency & {$[32]$} \\
\hline & & Salt sensitive & {$[26]$} \\
\hline \multirow[t]{4}{*}{$\Delta \operatorname{sigCDE}$} & \multirow[t]{4}{*}{ SigB, $2 \times$ that in $\mathrm{CS}$} & Resistant to $\mathrm{H}_{2} \mathrm{O}_{2}$ stress & {$[30]$} \\
\hline & & Salt tolerant & {$[25,26]$} \\
\hline & & Photoinhibition tolerant & {$[27,28]$} \\
\hline & & High carotenoid content & [27] \\
\hline \multirow[t]{5}{*}{$\Delta \operatorname{sigBCDE}$} & \multirow[t]{5}{*}{ None } & Sensitive to any oxidative stress & {$[30]$} \\
\hline & & Salt sensitive & {$[21]$} \\
\hline & & Bright light sensitive & {$[21]$} \\
\hline & & Heat sensitive & {$[21]$} \\
\hline & & Sensitive to nitrogen deficiency & [32] \\
\hline
\end{tabular}


of RNAP-SigC holoenzyme keeps cyanobacterial cells in the stationary phase [31, 32], while the SigE factor activates sugar catabolic reactions in darkness [21, 33, 34]. The SigD factor plays a major role in acclimation to bright light and singlet oxygen stresses [20, 30, 35, 36].

When three out of four group $2 \sigma$ factors are inactivated, the amount of the remaining group $2 \sigma$ factor is either doubled (if $\mathrm{SigB}$ or $\mathrm{SigD}$ is the remaining $\sigma$ factor), slightly enhanced (SigE), or remains similar (SigC) as in the control strain containing all $\sigma$ factors [30], see Table 1. As upregulation of either SigB or SigD offers extra protection against oxidative stress [30], it was tested if specific properties offered by group $2 \sigma$ factor inactivation strains would be beneficial for practical applications.

\section{Materials and Methods}

\section{Strains and Standard Growth Conditions}

The glucose-tolerant strain of Synechocystis sp. PCC 6803 was used as a control strain (CS) in these experiments and as a host strain for the construction of $\sigma$ factor inactivation strains [37]. Construction of the $\sigma$ factor inactivation strains $\Delta$ sigBCD, $\Delta$ sigBCE, $\Delta$ sigBDE, $\Delta$ sigCDE [37], and $\Delta$ sigBCDE [21] has been described earlier. If not otherwise stated, cells were grown in BG-11 medium supplemented with $20 \mathrm{mM}$ Hepes- $\mathrm{NaOH}$, pH 7.5, under constant illumination, photosynthetic photon flux density (PPFD) of $40 \mu \mathrm{mol} \mathrm{m} \mathrm{m}^{-2} \mathrm{~s}^{-1}$, at $32{ }^{\circ} \mathrm{C}$ in ambient air. For plates, agar (Bacto) was added $15 \mathrm{~g} / \mathrm{l}$, and plates for triple and quadruple mutants were supplemented with chloramphenicol (10 $\mu \mathrm{g} /$ $\mathrm{ml})$, kanamycin $(20 \mu \mathrm{g} / \mathrm{ml})$, spectinomycin $(10 \mu \mathrm{g} / \mathrm{ml})$, and streptomycin $(20 \mu \mathrm{g} / \mathrm{ml})$, and for quadruple mutant, also nourseothricin $(10 \mu \mathrm{g} / \mathrm{ml})$ was added. For growth experiments, $30 \mathrm{ml}$ cultures were grown in 100-ml Erlenmeyer flasks without antibiotics, in constant shaking at $90 \mathrm{rpm}$. Typically, $\mathrm{OD}_{730}$ was set to 0.1 (Genesys $10 \mathrm{~S}$ UV-VIS, Thermo Fisher Scientific, US) at the beginning of each experiment and growth was followed by measuring $\mathrm{OD}_{730}$. Before measurements, dense cultures were diluted so that $\mathrm{OD}_{730}$ did not exceed 0.4 and the dilutions were taken into account when the results were calculated.

Low-phosphate experiments were done by inoculating cells grown on BG-11 agar plates into BG-11-5\%P medium that contains only $8.75 \mu \mathrm{M} \mathrm{K}_{2} \mathrm{HPO}_{4}, 5 \%$ of that in the standard BG-11 medium.

\section{Consumption of Nitrate, Ammonium, and Phosphate}

To estimate ammonium, nitrate, or phosphate consumption, aliquots for measurements were taken every $24 \mathrm{~h}$. Cells were removed by filtering the culture trough $0.2 \mu \mathrm{m}$ cellulose acetate membrane filter (Whatman), and $\mathrm{NH}_{4}{ }^{+}, \mathrm{NO}_{3}{ }^{-}$, and $\mathrm{PO}_{4}{ }^{3-}$ were measured from the supernatant with Spectroquant ${ }^{\circledR}$ Ammonium, Nitrate, and Phosphate tests (Millipore) according to the manufacturer's instructions, respectively.

\section{KA2 Wastewater}

KA2 is contaminated underground water with anaerobic landfill leachate provided by Helsinki Region Environmental Services Authority HSY. Depending on the KA2 batch, it contained ammonium 5.9 to $6.9 \mathrm{mmol} / \mathrm{l}$ and phosphate 0.073 to $0.077 \mathrm{mmol} / \mathrm{l}$. The KA2 wastewater had a biochemical oxygen demand/chemical oxygen demand ratio of 0.055 (see Valev et al. [19]), which is the characteristic of "old" landfill leachate [38]. The high turbidity appearance of the KA2 wastewater indicated high total suspended solids that were removed by filtrating through a home-made granular activated carbon filter. Finally, wastewater was sterilized with $0.2 \mu \mathrm{m}$ PES membrane bottle-top filter (VWR, USA) prior to the experiment. For some experiments, KA2 was supplemented with phosphate to reach the same level as in the BG-11 medium, and for some experiments, the KA2 was also buffered with $20 \mathrm{mM}$ Hepes, $\mathrm{pH} 7.5$, as indicated.

\section{High-Light Stress}

High-light stress conditions were applied in Multicultivator MC1000 device (PSI, Czech Republic). The device consist of eight experimental tubes, submersed in a water bath that maintains the temperature at $32{ }^{\circ} \mathrm{C}$. Cultures were mixed by bubbling with air (circa $60 \mathrm{ml} / \mathrm{min}$ ), provided by MC1000's factory supplied air pump. Light conditions for each tube were adjusted to the PPFD of $500 \mu \mathrm{mol} \mathrm{m}{ }^{-2} \mathrm{~s}^{-1}$ with a submersible light sensor (Model: US-SQS/L, Heinz Walz, Germany). An OD sensor at $730 \mathrm{~nm}$ inside each tube was used to monitor growth every $30 \mathrm{~min}$.

\section{Results}

\section{Synechocystis sp. PCC 6803 Cells are Efficient in Acquisition of Nutrients from Growth Medium}

To get an idea on how rapidly the cyanobacterium Synechocystis sp. PCC 6803 cells acquire nutrients from the growth medium, the glucose-tolerant control strain was grown in BG-11 medium under constant illumination (PPFD $40 \mu \mathrm{mol} \mathrm{m}^{-2} \mathrm{~s}^{-1}$ ), at $32{ }^{\circ} \mathrm{C}$ in $\mathrm{CO}_{2}$-enriched atmosphere, and consumption of nitrate and phosphate was followed for a few days. BG-11 is a rich medium containing $17.6 \mathrm{mM}$ nitrate and $175 \mu \mathrm{M}$ phosphate. Phosphate was rapidly consumed and only $2 \%$ of phosphate remained in the growth medium after four days (Fig. 1), while $\sim 50 \%$ of nitrate had been 
consumed at that point. However, rapid growth of cells continued for a week, indicating that acquisition of phosphorus exceeds that needed for growth, and extra phosphorus was stored for future utilization (Fig. 1). Cells continued growth for the whole 13 days of this experiment, but the growth was slow after the first 8 days.

\section{Landfill Leachate Wastewater is Usable as a Batch Growth Medium for Synechocystis sp. PCC 6803}

As Synechocystis cells removed nutrients efficiently from the BG-11 medium, it was next tested how well Synechocystis sp. PCC 6803 cells removed nutrients from landfill leachate wastewater KA2 in these standard growth conditions. The KA2 wastewater is poor in nitrate but rich in ammonium (5.9-6.9 $\mathrm{mM}$ depending on the batch), and, therefore, the amount of available nitrogen in KA2 is $34-39 \%$ of that in BG-11 medium. The phosphate concentration of KA2 was $41-44 \%$ of that in BG-11 medium. When the control strain cells were grown under constant illumination (PPFD $40 \mu \mathrm{mol} \mathrm{m}{ }^{-2} \mathrm{~s}^{-1}$ ), at $32{ }^{\circ} \mathrm{C}$ in KA2 medium in ambient air, only traces of ammonium or phosphate remained in KA after three days (Fig. 2), but cells continued to grow longer, indicating that cyanobacteria store nutrients also from wastewater (Fig. 3).

In addition to the control strain, the growth of the collection of group $2 \sigma$ factor mutant strains in KA2 was also tested (Fig. 3). These strains are of interest because

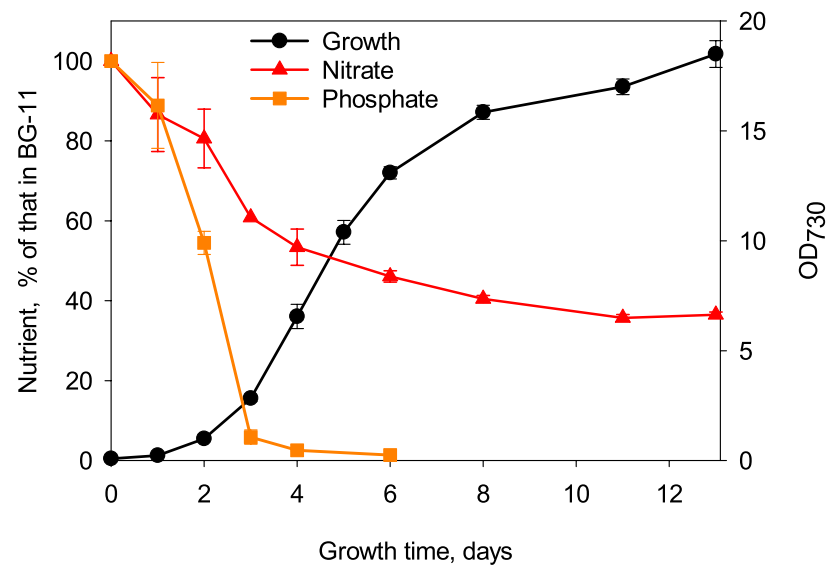

Fig. 1 Growth and uptake of nitrate and phosphate from BG-11 growth medium. The growth of Synechocystis culture was monitored once a day by measuring $\mathrm{OD}_{730}$, and the amounts of remaining nitrate and phosphate in BG-11 growth medium were also measured daily. The nitrate concentration of BG-11 is $17.6 \mathrm{mM}$ and phosphate concentration is $0.175 \mathrm{mM}$. The $\mathrm{pH}$ of the BG-11 growth medium was buffered by adding $20 \mathrm{mM}$ Hepes- $\mathrm{NaOH}, \mathrm{pH} 7.5$, and the control strain cells were grown under constant illumination, PPFD $40 \mu \mathrm{mol} \mathrm{m} \mathrm{m}^{-2} \mathrm{~s}^{-1}$, at $32{ }^{\circ} \mathrm{C}$. The growth chamber air was enriched with $3 \% \mathrm{CO}_{2}$. Three independent biological replicates were measured, and the error bars denote SE
$\Delta$ sigCDE and $\Delta$ sigBCE strains grow better than the control strain in some stress conditions like chemically induced oxidative stress, whereas the $\triangle$ sigBCDE strain is vulnerable to all stress conditions $[21,30]$. All $\sigma$ factor triple inactivation strains grow like the control strain in BG-11 medium for 10 days in standard growth conditions [37] and the quadruple mutant $\Delta$ sigBCDE almost as well (Supplemental Fig S1). For a comparison of KA2 and BG-11, the control strain was also grown in BG-11 medium.

All strains grew similarly in wastewater as in the BG-11 growth medium for four days (Fig. 3a). The finding that the extremely stress-sensitive $\Delta$ sigBCDE strain $[21,32,39]$ grew like the control strain for a few days indicates that the KA2 wastewater is devoid of compounds that are immediately toxic to Synechocystis sp. PCC 6803 or induce substantial oxidative stress. However, although KA2 wastewater permitted normal growth for a few days, cells grew faster in BG-11 medium than those in KA2 after the first 6 days and reached a higher cell density (Fig. 3a). This suggests that long-term growth in KA2 is a stress for Synechocystis sp. PCC 6803 . After 5 days, the $\Delta$ sigBCDE strain lost the ability to grow (Fig. 3a) and bleaching of pigments became visible after 8 days (Fig. 3b-d). The pale yellow-orange color of the old $\triangle$ sigBCDE culture indicated that almost all blue phycobilin pigments and the yellow-green chlorophyll $a$ pigments had been degraded, and only some carotenoids remained (Fig. 3d), indicating that specific changes in gene expression are required for long-term growth of Synechocystis sp. PCC 6803 in KA2 medium, and this acclimation does not function without group $2 \sigma$ factors.

In KA2 wastewater, none of the mutant strains was superior compared to the control strain. $\triangle$ sigBDE (contains

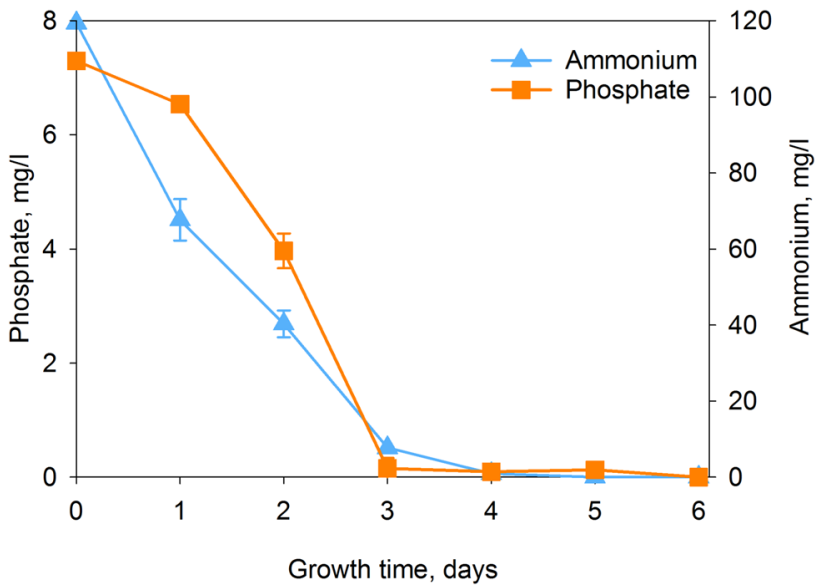

Fig. 2 Uptake of ammonium and phosphate from KA2 wastewater. The control strain of Synechocystis was grown in KA2 wastewater under constant illumination, PPFD $40 \mu \mathrm{mol} \mathrm{m} \mathrm{m}^{-2} \mathrm{~s}^{-1}$, at $32{ }^{\circ} \mathrm{C}$ in ambient air. The original ammonium concentration of KA2 wastewater was $6.9 \mathrm{mM}(119 \mathrm{mg} / \mathrm{ml})$ and phosphate was $0.077 \mathrm{mM}$ $(7.3 \mathrm{mg} / \mathrm{l})$, whereas the nitrate concentration was negligible 


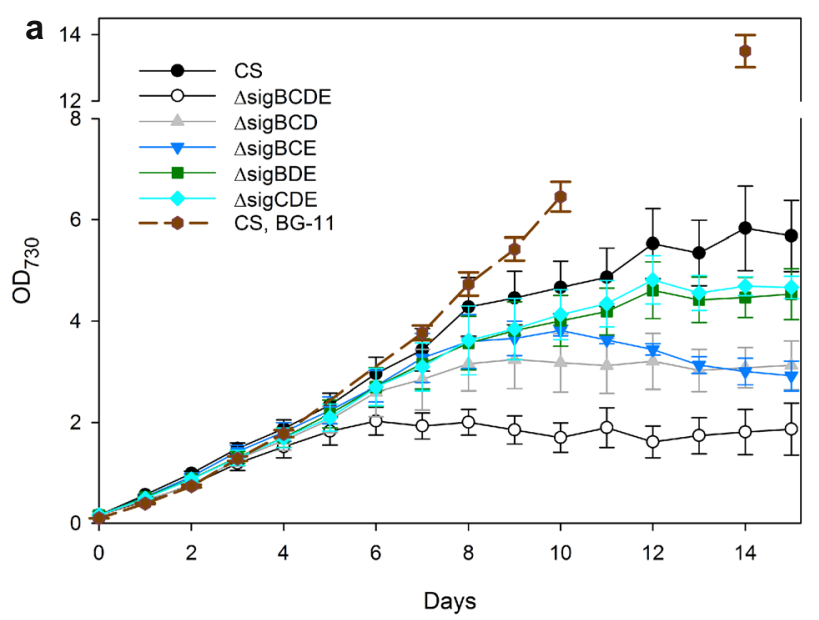

b

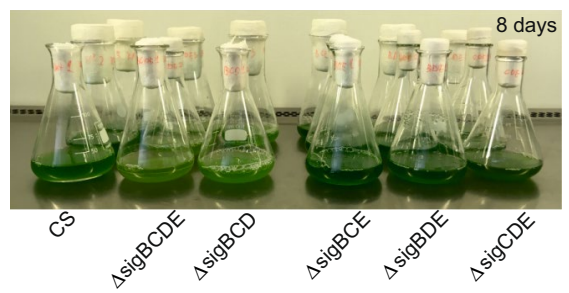

C

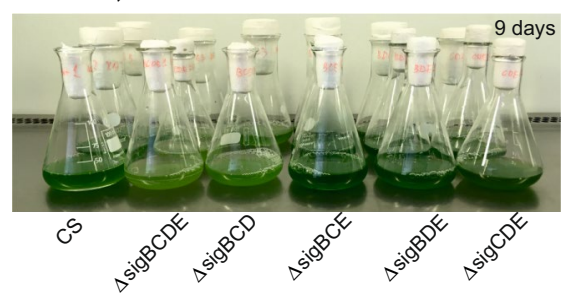

d

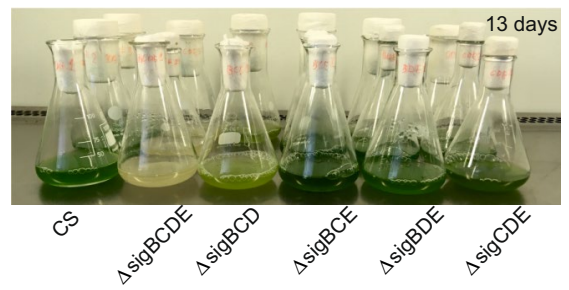

Fig. 3 Growth of the control and $\sigma$ factor mutant strains in wastewater. a $\mathrm{OD}_{730}$ was set to 0.1 and the growth was followed for 15 days by measuring $\mathrm{OD}_{730}$ once a day. For a control experiment, control strain cells were grown in standard BG-11 growth medium. The cells were grown under constant illumination, PPFD $40 \mu \mathrm{mol} \mathrm{m} \mathrm{m}^{-2} \mathrm{~s}^{-1}$, at $32{ }^{\circ} \mathrm{C}$ in ambient air. Three independent biological replicates were measured, and the error bars denote SE. The wastewater cultures were photographed after 8 (b), $9(\mathbf{c})$, and 13 (d) days

only $\mathrm{SigC}$ ) and $\Delta \operatorname{sigCDE}$ (contains only $\mathrm{SigB}$ ) continued growth for 11 days just like the control strain, and after the 12th day, the cell content of these strains remained constant and no bleaching was observed (Fig. 3). The cessation of growth occurred already after 8 days for strains $\triangle$ sigBCD and $\triangle$ sigBCE. The $\triangle$ sigBCE strain did not show any bleaching, whereas $\Delta \operatorname{sigBCD}$ cells turned yellowish-green,

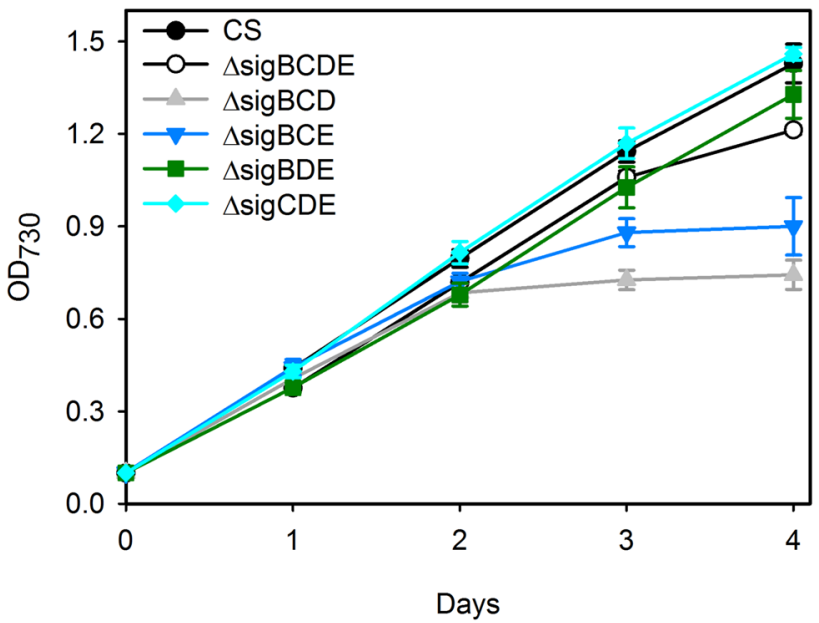

Fig. 4 Growth of the control and mutant cells in low-phosphate conditions. The $\mathrm{OD}_{730}$ was set to 0.1 , and cell cultures were grown in BG-11 medium containing only $5 \%$ of the normal phosphate concentration of BG-11 $(8.75 \mu \mathrm{M})$. The growth medium was buffered with $20 \mathrm{mM}$ Hepes-NaOH, pH 7.5. Cells were grown under constant illumination, PPFD $40 \mu \mathrm{mol} \mathrm{m}{ }^{-2} \mathrm{~s}^{-1}$, at $32{ }^{\circ} \mathrm{C}$ in ambient air. Three independent biological replicates were measured for each strain. The error bars denote SE

indicating bleaching of blue phycobilin pigments in the stationary phase (Fig. 3d).

Phosphate transporter genes are highly upregulated at transcriptional level in normal phosphate-replete BG-11 medium in the $\triangle$ sigBCD strain; the data shown in Supplemental Table S1 have been collected from previously published DNA microarray results ( [29], Geo accessions GSE117478, GSE50060 and GSE69981). The initial phosphate concentration of the wastewater was $41-44 \%$ of that in standard BG-11 medium, which raises the question whether phosphate limitation is likely the cause for the observed early entry of cells to the stationary phase in KA2 wastewater. For this, the growth of the Synechocystis sp. PCC6803 strains in BG-11-5\%P medium that contains only $8.75 \mu \mathrm{M}$ phosphate was tested (Fig. 4). The control strain grew well in BG-11-5\%P medium for the tested four days. All mutant strains grew as well as the control strain for the first 2 days in BG-11-5\%P (Fig. 4), indicating that they all were able to harness phosphate even when phosphate concentration was low. The growth of $\triangle \operatorname{sigBCD}$ and $\triangle \operatorname{sigBCE}$ strains decelerated considerably in BG-11-5\% P medium (Fig. 4). As the quadruple mutant $\Delta$ sigBCDE grew well in BG-11-5\%P medium, the results indicate that low phosphate sensitivity is not due to missing $\sigma$ factors but caused by a combined effect of missing $\sigma$ factors and overdose of the remaining group $2 \sigma$ factor (Table 1). To directly test if the low phosphate content of KA2 was the reason for the early cessation of growth in KA2, phosphate was added to KA2 so that the final phosphate concentration of KA2 was the same 
as in the BG-11 medium. However, this addition did not improve the growth of $\triangle \operatorname{sigBCD}$ or $\triangle$ sigBCE strains, and actually $\Delta$ sigBCD cells had a long lag phase before they started to grow (Fig. 5a), indicating that low phosphate was not the main reason for early cessation of the growth of the $\Delta$ sigBCD or $\Delta$ sigBCE strains in KA2.

Unlike in typical growth experiments, KA2 wastewater was not buffered with $20 \mathrm{mM}$ Hepes, $\mathrm{pH} 7.5$, as expensive buffers could not be used in real wastewater treatments. When cyanobacteria are grown in ambient air without buffering, the $\mathrm{pH}$ of growth medium increases rapidly (Fig. 5b). In ammonium containing wastewaters, high $\mathrm{pH}$ would lead to conversion of ammonium to toxic volatile ammonia. To test consequences of that, the growth and nutrient uptake of CS, $\Delta$ sigBCD, and $\Delta$ sigBCE cells in wastewater supplemented with phosphate and buffered with $20 \mathrm{mM}$ Hepes to $\mathrm{pH} 7.5$ were measured. The removal of ammonium from KA2 after buffering (Fig. 5c) was slower than without buffering (Fig. 2), indicating that without buffering, volatile ammonia was escaping. No differences were detected in ammonium removal between CS, $\Delta$ sigBCD, and $\Delta$ sigBCE cells (Fig. $5 \mathrm{c}$ ). The $\Delta$ sigBCE strain first grew like CS in buffered KA2 and growth was even better than that of the CS in the end of the experiment (Fig. 5c). Rapid growth of $\Delta$ sigBCE cells in the end of the experiment was accompanied with more rapid removal of phosphate than that in the control strain (Fig. 5c). Contrary to the $\Delta$ sigBCE strain, $\Delta$ sigBCD cells grew more slowly than CS cells even after buffering KA2 medium and $\triangle$ sigBCD cells collected phosphate more slowly from buffered KA2 than the other strains (Fig. 5c).

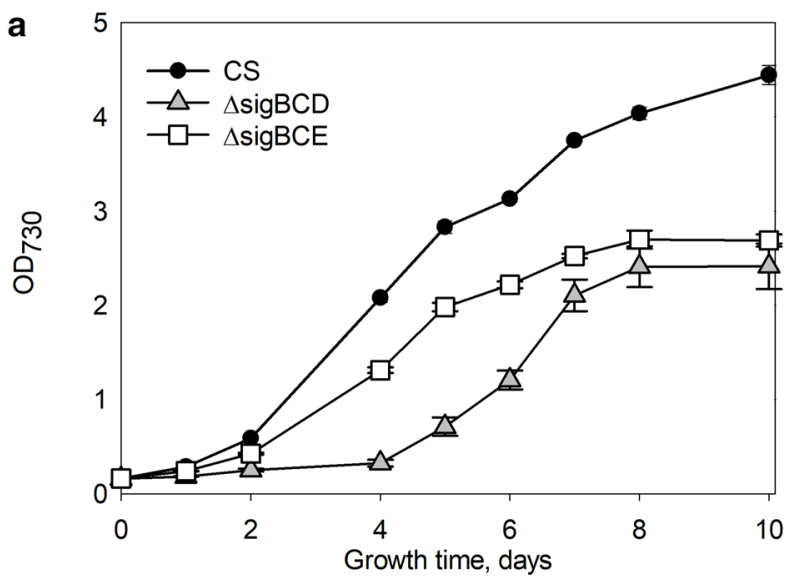

C

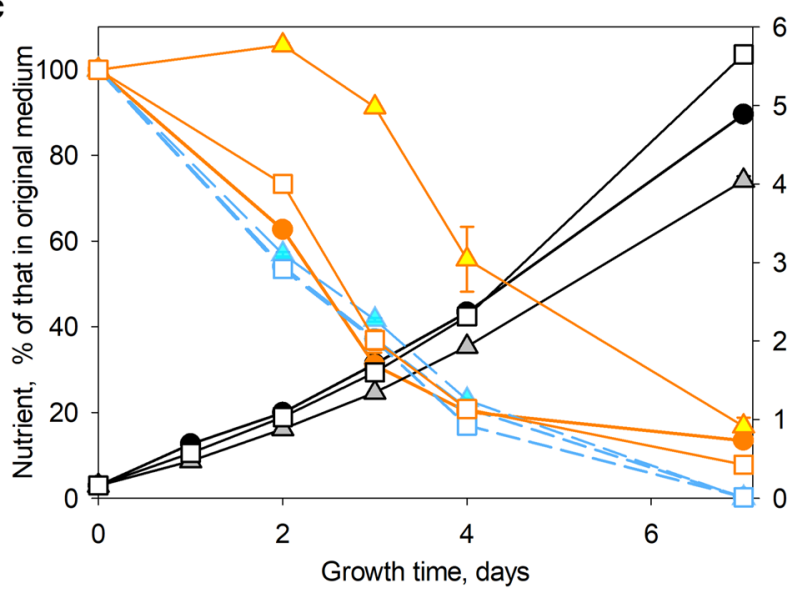

Fig. 5 Effects of phosphate and $\mathrm{pH}$ on growth and nutrient removal. a The control, $\Delta$ sigBCD, and $\Delta$ sigBCE strains of Synechocystis were grown in KA2 wastewater supplemented with BG-11 level phosphate under constant illumination, PPFD $40 \mu \mathrm{mol} \mathrm{m} \mathrm{m}^{-2} \mathrm{~s}^{-1}$, at $32{ }^{\circ} \mathrm{C}$ in ambient air. b The control strain of Synechocystis was grown in BG-11 medium without buffering under constant illumination, PPFD $40 \mu \mathrm{mol} \mathrm{m} \mathrm{m}^{-2} \mathrm{~s}^{-1}$, at $32{ }^{\circ} \mathrm{C}$ in ambient air, and cell density and $\mathrm{pH}$

6
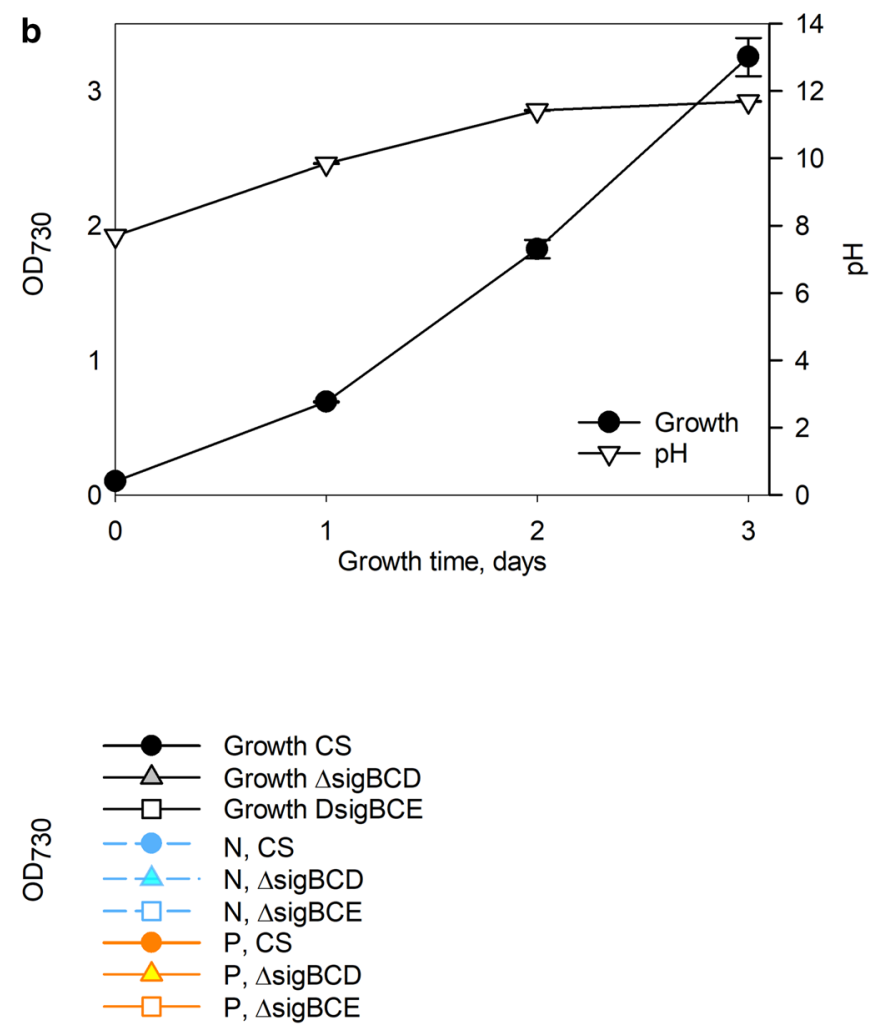

of growth medium were measured. c The control, $\triangle$ sigBCD, and $\Delta$ sigBCE strains of Synechocystis were grown in KA2 wastewater supplemented with BG-11 level phosphate and $20 \mathrm{mM}$ Hepes, $\mathrm{pH}$ 7.5 , under constant illumination, PPFD $40 \mu \mathrm{mol} \mathrm{m} \mathrm{m}^{-2} \mathrm{~s}^{-1}$, at $32{ }^{\circ} \mathrm{C}$ in ambient air. Three independent biological replicates were measured. The error bars denote SE 


\section{$\Delta$ sigBCE Grows Rapidly in High Light in a Laboratory-scale Bioreactor}

An optimal cyanobacterial strain that would be utilized in wastewater treatment should tolerate not only nutrient imbalance or harmful substances but also non-optimal temperature and light conditions. The wastewater tests were done in standard laboratory condition. However, cyanobacteria utilized to treat wastewater can be expected to be exposed to brighter light than this laboratory standard, PPFD $40 \mu \mathrm{mol} \mathrm{m} \mathrm{m}^{-2} \mathrm{~s}^{-1}$. Next the ability of $\sigma$ factor inactivation strains was tested to cope with high light in otherwise standard conditions. The growth in bright light was tested in a laboratory-scale bioreactor, "Multicultivator." The strain $\Delta$ sigBCE that contains only the SigD factor grew better than the control strain at PPFD $500 \mu \mathrm{mol} \mathrm{m}^{-2} \mathrm{~s}^{-1}$, and the slowest growth was measured for the $\triangle$ sigBCDE strain (Fig. 6). Thus, utilization of the $\Delta$ sigBCE strain might be useful if cells can be expected to experience high light.

\section{Discussion}

The KA2 wastewater was found to be suitable for Synechocystis sp. PCC 6803 (Fig. 3). The group $2 \sigma$ factor less $\triangle$ sigBCDE strain is sensitive for many stress conditions, including oxidative stress (Table 1), and can therefore be considered as a stress-sensitive indicator strain. The growth of $\triangle \operatorname{sigBCDE}$ in KA2 for few days indicates that KA2 does not induce immediate oxidative stress to Synechocystis sp. PCC 6803 cells (Fig. 3). The growth of cyanobacteria in KA2 wastewater removed almost all ammonium and phosphate within three days (Fig. 2). The removal of ammonium

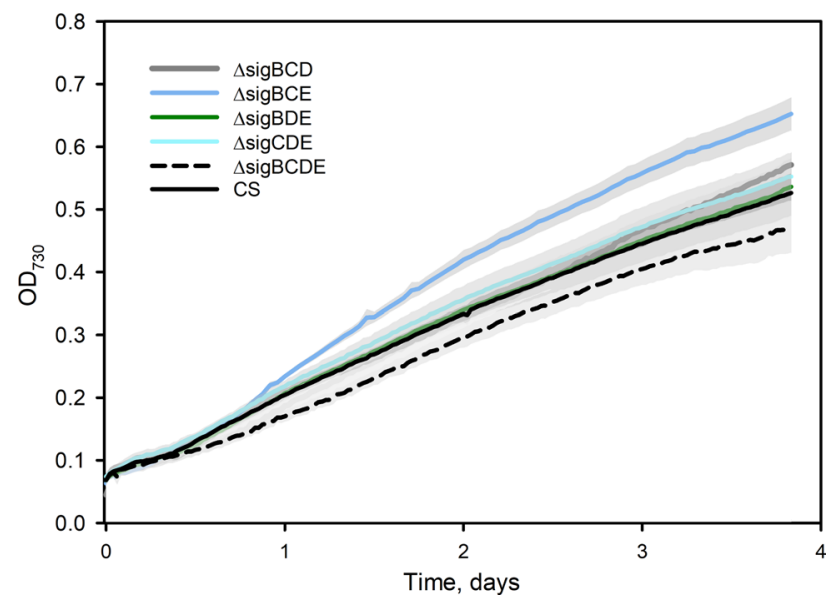

Fig. 6 Growth of the control and $\sigma$ factor inactivation strains in continuous bright light, PPFD $500 \mu \mathrm{mol} \mathrm{m} \mathrm{m}^{-2} \mathrm{~s}^{-1}$. Cells were grown in a Multicultivator. Lines represent average values from at least three independent measurements, and the grey areas show SE was slower if wastewater was buffered to 7.5 (Fig. 5c), indicating that part of the ammonium was released as ammonia in un-buffered wastewater and was not utilized by cyanobacteria. Cultivation of cyanobacteria in un-buffered medium under ambient air causes a rapid increase of pH (Fig. 5b), which facilitates conversion of ammonium to ammonia. Instead of using expensive buffers to keep the $\mathrm{pH}$ of the growth medium at the desired value, automatic addition of $\mathrm{CO}_{2}$ is used to counteract the growth-induced increase of $\mathrm{pH}$ in bioreactors [40]. It is shown that if growth chamber air is supplemented with $3 \% \mathrm{CO}_{2}$, Synechocystis sp. PCC6803 cells grow very fast and the pH of un-buffered BG-11 remains 8.2 [9]. Thus, wastewaters containing ammonium might be possible to remediate with cyanobacteria in a $\mathrm{pH}$-controlled process. For wastewaters containing nitrate as a nitrogen source, $\mathrm{pH}$ is not as critical. The results show that cyanobacteria are able to remove $90 \%$ of ammonium and phosphate within few days from wastewater, and they are also able to collect nitrate efficiently, taking into account that the nitrate concentration of BG-11 medium is $17.6 \mathrm{mM}$.

Cyanobacteria prefer ammonium as a nitrogen source $[5,41]$, and a regulatory network including the amounts of 2-oxoglutarate and ATP, and the regulatory proteins PII, PipX, and NtcA orchestrates nitrogen metabolism according to the $\mathrm{C} / \mathrm{N}$ ratio, for a review see [8]. In nitrogen deficiency, abundant blue phycobiliproteins are degraded, followed by slow decrease of the yellow-green chl $a$ and strong accumulation of protective carotenoids, which give the typical yellowish-brown color to cyanobacterial cultures in nitrogen limitation [32]. The wastewater cultures of the CS strain remained green throughout the whole experiment (Fig. 3) although growth was arrested earlier than in BG-11 medium, indicating that nitrogen deficiency might not be the main reason for early growth cessation.

Synechocystis sp. PCC cells consumed most of the phosphate of the standard BG-11 medium or KA2 wastewater within 3 days but grew well in BG-11-5\% P for four days. Thus, phosphate deprivation is unlikely to be the main reason for the cessation of growth after six days in wastewater containing 8 times more phosphate than in BG-11-5\%P. In cyanobacteria, phosphate taken from growth medium is deposited to an inner cellular pool and to an extracellular pool [42-44]. Cells first both take in phosphate and adsorb it with the extracellular matrix, and when the growth medium is depleted of phosphate, phosphate from the extracellular matrix is taken in [44]. Inside the cells, phosphate is stored as polyphosphate [17], and Synechocystis has been speculated to use multiple DNA copies as a phosphate storage as well, as at least the number of genome copies is dependent on the amount of phosphate [45].

The high expression of phosphate transporters in the $\Delta$ sigBCD strain in these standard growth conditions (Supplemental Table S1) and the early growth-arrest phenotype 
of $\Delta$ sigBCD in low-phosphate medium (Fig. 4) may indicate that $\Delta$ sigBCD has defects either in acquisition or in utilization of phosphate, and slower removal of phosphate by $\Delta$ sigBCD (Fig. 5c) indeed points to abnormalities in phosphate acquisition. The defects in phosphate acquisition/utilization are connected to the combined effects of missing SigB, SigC, and SigD factors and overdose of SigE factor (Table 1) as $\Delta$ sigBCDE strain missing all group $2 \sigma$ factors do not overexpress phosphate transporter genes (Supplemental Table S1) and grow better than $\triangle$ sigBCD in low-phosphate medium (Fig. 4). Expression of phosphate transporter genes is normal also in a SigE overexpression strain that contains all group $2 \sigma$ factors [33]. However, addition of extra phosphate did not improve growth of $\triangle$ sigBCD in KA2 (Fig. 5a). After buffering the KA2 wastewater (Fig. 5c), the long lag phase of $\Delta$ sigBCD strain shown in Fig. 5a disappeared. The ammonium concentrations of KA2 batch were $6.9 \mathrm{mM}$ as shown in Fig. 5 and $5.9 \mathrm{mM}$ as shown in Fig. 2, suggesting that toxic ammonia was formed in higher quantity as shown in Fig. 5a than that shown in Fig. 2a. Thus, it is possible that in addition to having a slowly functioning phosphate uptake system, the $\triangle$ sigBCD strain is also more sensitive to ammonia than CS. That would not be surprising, as both main oxidative stress responsive $\sigma$ factors, $\mathrm{SigB}$ and SigD, are missing in $\triangle$ sigBCD [30].

Contrary to $\Delta$ sigBCD, the $\Delta$ sigBCE strain showed normal expression of phosphate transporter genes when grown in standard BG-11 medium. Unfortunately, transcriptome analysis of $\Delta$ sigBCE in standard conditions (Hakkila et al. 2019) does not give direct hints for possible mechanism(s), as the majority of up- or down-regulated genes produce proteins with unknown functions. Interestingly, $\mathrm{pH}$ adjustment of wastewater to 7.5 improved the growth of $\Delta$ sigBCE, and actually $\triangle$ sigBCE cells removed more phosphate and had higher cell density than the control strain in the end of the experiment (Fig. 5c). The $\Delta$ sigBCE strain that contains a high amount of the SigD factor grows better than the control strain also in high light (Fig. 6), singlet oxygen, or $\mathrm{H}_{2} \mathrm{O}_{2}$ stresses [30]. Thus, $\Delta$ sigBCE strain is potentially interesting for wastewater treatment as high light and reactive oxygen species tolerance might provide extra robustness required for wastewater treatment outside the strictly controlled laboratory conditions.

Cyanobacteria have already been tested for wastewater bioremediation, and the results are encouraging. A native Synechocystis sp. from Costa Rica was isolated and then nutrient removal was tested from synthetic medium containing same levels of phosphorus and nitrogen $(95 \mu \mathrm{M}$ phosphate and $3.9 \mathrm{mM}$ ammonium) as Rio Azul and San José. Synechocystis sp. removed $70 \%$ of phosphate and $30 \%$ of ammonium within 5 days, however, green alga Chlorella was more efficient in removing both nutrients almost completely [46]. Synechocystis sp. was shown to remove phosphate in a recirculating aquaculture system [47], and other cyanobacterial species were shown to reduce the nitrogen content of dairy wastewater by circa $80 \%$ [48]. In accordance with these results, these results suggest that cyanobacteria might offer a potential solution for nitrogen and phosphorus removal from wastewaters.

Acknowledgements Open access funding provided by University of Turku (UTU) including Turku University Central Hospital. This work was supported by the Academy of Finland (265807 to T.T. and 307335 to E.T.), NordForsk (NordAqua project), the University of Turku Graduate School, Novo Nordisk Foundation (T.T) and Helsinki Region Environmental Services Authority HSY.

\section{Compliance with Ethical Standards}

Conflict of interest: The authors declare that they have no conflict of interest.

Open Access This article is licensed under a Creative Commons Attribution 4.0 International License, which permits use, sharing, adaptation, distribution and reproduction in any medium or format, as long as you give appropriate credit to the original author(s) and the source, provide a link to the Creative Commons licence, and indicate if changes were made. The images or other third party material in this article are included in the article's Creative Commons licence, unless indicated otherwise in a credit line to the material. If material is not included in the article's Creative Commons licence and your intended use is not permitted by statutory regulation or exceeds the permitted use, you will need to obtain permission directly from the copyright holder. To view a copy of this licence, visit http://creativecommons.org/licenses/by/4.0/.

\section{References}

1. Delrue F, Álvarez-Diaz PA, Fon-Sing S, Fleury G, Sassi J (2016) The environmental biorefinery: using microalgae to remediate wastewater, a win-win paradigm. Energies 9:132

2. Pittman JK, Dean AP, Osundeko O (2011) The potential of sustainable algal biofuel production using wastewater resources. Bioresour Technol 102:17-25

3. Renuka N, Sood A, Prasanna R, Ahluwalia AS (2015) Phytoremediation of wastewaters: a synergistic approach using microalgae for bioremediation and biomass generation. Int J Environ Sci Technol 12:1443-1460

4. Flores E, Frias JE, Rubio LM, Herrero A (2005) Photosynthetic nitrate assimilation in cyanobacteria. Photosynth Res 83:117-133

5. Muro-Pastor MI, Reyes JC, Florencio FJ (2005) Ammonium assimilation in cyanobacteria. Photosynth Res 83:135-150

6. Valladares A, Montesinos ML, Herrero A, Flores E (2002) An ABC-type, high-affinity urea permease identified in cyanobacteria. Mol Microbiol 43:703-715

7. Montesinos ML, Muro-Pastor AM, Herrero A, Flores E (1998) Ammonium/methylammonium permeases of a cyanobacterium. Identification and analysis of three nitrogen-regulated amt genes in Synechocystis sp. PCC 6803. J Biol Chem 273:31463-31470

8. Esteves-Ferreira AA, Inaba M, Fort A, Araújo WL, Sulpice R (2018) Nitrogen metabolism in cyanobacteria: metabolic and molecular control, growth consequences and biotechnological applications. Crit Rev Microbiol 44:541-560 
9. Kurkela J, Hakkila K, Antal T, Tyystjärvi T (2017) Acclimation to high $\mathrm{CO}_{2}$ requires the $\omega$ subunit of the RNA polymerase in Synechocystis. Plant Physiol 174:172-184

10. Drath M, Kloft N, Batschauer A, Marin K, Novak J, Forchhammer K (2008) Ammonia triggers photodamage of photosystem II in the cyanobacterium Synechocystis sp. strain PCC 6803. Plant Physiol 147:206-215

11. Watzer B, Forchhammer K (2018) Cyanophycin synthesis optimizes nitrogen utilization in the unicellular cyanobacterium Synechocystis sp. PCC 6803. Appl Environ Microbiol 84:e01298-e1318

12. Pitt FD, Mazard S, Humphreys L, Scanlan DJ (2010) Functional characterization of Synechocystis sp. strain PCC 6803 pst 1 and pst2 gene clusters reveals a novel strategy for phosphate uptake in a freshwater cyanobacterium. J Bacteriol 192:3512-3523

13. Burut-Archanai S, Eaton-Rye JJ, Incharoensakdi A (2011) $\mathrm{Na}^{+}$-stimulated phosphate uptake system in Synechocystis sp. PCC 6803 with Pst1 as a main transporter. BMC Microbiol 11:225

14. Gómez-García MR, Losada M, Serrano A (2003) Concurrent transcriptional activation of $p p a$ and $p p x$ genes by phosphate deprivation in the cyanobacterium Synechocystis sp. strain PCC 6803. Biochem Biophys Res Commun 302:601-609

15. Suzuki S, Ferjani A, Suzuki I, Murata N (2004) The SphS-SphR two component system is the exclusive sensor for the induction of gene expression in response to phosphate limitation in Synechocystis. J Biol Chem 279:13234-13240

16. Hirani TA, Suzuki I, Murata N, Hayashi H, Eaton-Rye JJ (2001) Characterization of a two-component signal transduction system involved in the induction of alkaline phosphatase under phosphate-limiting conditions in Synechocystis sp. PCC 6803. Plant Mol Biol 45:133-144

17. Morohoshi T, Maruo T, Shirai Y, Kato J, Ikeda T, Takiguchi N, Ohtake H, Kuroda A (2002) Accumulation of inorganic polyphosphate in phoU mutants of Escherichia coli and Synechocystis sp. strain PCC6803. Appl Environ Microbiol 68:4107-4110

18. Michels MH, Vaskoska M, Vermue MH, Wijffels RH (2014) Growth of Tetraselmis suecica in a tubular photobioreactor on wastewater from a fish farm. Water Res 65:290-296

19. Valev D, Santos HS, Tyystjärvi E (2019) Reliable and stable wastewater treatment Neochloris oleoabundensis in a tubular bioreactor. J Appl Phycol in press.

20. Imamura S, Yoshihara S, Nakano S, Shiozaki N, Yamada A, Tanaka K, Takahashi H, Asayama M, Shirai M (2003) Purification, characterization, and gene expression of all sigma factors of RNA polymerase in a cyanobacterium. J Mol Biol 325:857-872

21. Koskinen S, Hakkila K, Gunnelius L, Kurkela J, Wada H, Tyystjärvi $\mathrm{T}$ (2016) In vivo recruitment analysis and a mutant strain without any group $2 \sigma$ factor reveal roles of different sigma factors in cyanobacteria. Mol Microbiol 99:43-54

22. Stensjö K, Vavitsas K, Tyystjärvi T (2018) Harnessing transcription for bioproduction in cyanobacteria. Physiol Plant 162:148-155

23. Tuominen I, Pollari M, Tyystjärvi E, Tyystjärvi T (2006) The SigB $\sigma$ factor mediates high-temperature responses in the cyanobacterium Synechocystis sp. PCC6803. FEBS Lett 580:319-323

24. Singh AK, Summerfield TC, Li H, Sherman LA (2006) The heat shock response in the cyanobacterium Synechocystis sp. strain PCC 6803 and regulation of gene expression by HrcA and SigB. Arch Microbiol 186:273-286

25. Nikkinen HL, Hakkila K, Gunnelius L, Huokko T, Pollari M, Tyystjärvi T (2012) The SigB $\sigma$ factor regulates multiple salt acclimation responses of the cyanobacterium Synechocystis $\mathrm{sp}$. PCC 6803. Plant Physiol 158:514-523
26. Tyystjärvi T, Huokko T, Rantamäki S, Tyystjärvi E (2013) Impact of different group 2 sigma factors on light use efficiency and high salt stress in the cyanobacterium Synechocystis sp. PCC 6803. PLoS ONE 8:e63020

27. Hakkila K, Antal T, Gunnelius L, Kurkela J, Matthijs HCP, Tyystjärvi E, Tyystjärvi T (2013) Group 2 sigma factor mutant $\Delta$ sigCDE of the cyanobacterium Synechocystis sp. PCC 6803 reveals functionality of both carotenoids and flavodiiron proteins in photoprotection of photosystem II. Plant Cell Physiol 54:1827-1838

28. Hakkila K, Antal T, Rehman AU, Kurkela J, Wada H, Vass I, Tyystjärvi E, Tyystjärvi T (2014) Oxidative stress and photoinhibition can be separated in the cyanobacterium Synechocystis sp. PCC 6803. Biochim Biophys Acta 1837:217-225

29. Kaczmarzyk D, Anfelt J, Särnegrim A, Hudson EP (2014) Overexpression of sigma factor SigB improves temperature and butanol tolerance of Synechocystis sp. PCC6803. J Biotechnol 182-183:54-60

30. Hakkila K, Valev D, Antal T, Tyystjärvi E, Tyystjärvi T (2019) Group 2 sigma factors are central regulators of oxidative stress acclimation in cyanobacteria. Plant Cell Physiol 60:436-447

31. Asayama M, Imamura S, Yoshihara S, Miyazaki A, Yoshida N, Sazuka T, Kaneko T, Ohara O, Tabata S, Osanai T et al (2004) $\mathrm{SigC}$, the group 2 sigma factor of RNA polymerase, contributes to the late-stage gene expression and nitrogen promoter recognition in the cyanobacterium Synechocystis sp. strain PCC 6803. Biosci Biotechnol Biochem 68:477-487

32. Antal T, Kurkela J, Parikainen M, Kårlund A, Hakkila K, Tyystjärvi E, Tyystjärvi T (2016) Roles of group 2 sigma factors in acclimation of the cyanobacterium Synechocystis sp. PCC 6803 to nitrogen deficiency. Plant Cell Physiol 57:1309-1318

33. Osanai T, Oikawa A, Azuma M, Tanaka K, Saito K, Hirai MY, Ikeuchi M (2011) Genetic engineering of group $2 \sigma$ factor SigE widely activates expressions of sugar catabolic genes in Synechocystis species PCC 6803. J Biol Chem 286:30962-30971

34. Osanai T, Kanesaki Y, Nakano T, Takahashi H, Asayama M, Shirai M, Kanehisa M, Suzuki I, Murata N, Tanaka K (2005) Positive regulation of sugar catabolic pathways in the cyanobacterium Synechocystis sp. PCC 6803 by the group 2 sigma factor SigE. J Biol Chem 280:30653-30659

35. Pollari M, Gunnelius L, Tuominen I, Ruotsalainen V, Tyystjärvi E, Salminen T, Tyystjärvi T (2008) Characterization of single and double inactivation strains reveals new physiological roles for group $2 \sigma$ factors in the cyanobacterium Synechocystis sp. PCC 6803. Plant Physiol 147:1994-2005

36. Pollari M, Ruotsalainen V, Rantamäki S, Tyystjärvi E, Tyystjärvi T (2009) Simultaneous inactivation of sigma factors $\mathrm{B}$ and D interferes with light acclimation of the cyanobacterium Synechocystis sp. strain PCC 6803. J Bacteriol 191:3992-4001

37. Pollari M, Rantamäki S, Huokko T, Kårlund-Marttila A, Virjamo V, Tyystjärvi E, Tyystjärvi T (2011) Effects of deficiency and overdose of group 2 sigma factors in triple inactivation strains of Synechocystis sp. strain PCC 6803. J Bacteriol 193:265-273

38. Renou S, Givaudan JG, Poulain S, Dirassouyan F, Moulin P (2008) Landfill leachate treatment: Review and opportunity. J Hazard Mater 150:468-493

39. Koskinen S, Hakkila K, Kurkela J, Tyystjärvi E, Tyystjärvi T (2018) Inactivation of group $2 \sigma$ factors upregulates production of transcriptional and translational machineries in the cyanobacterium Synechocystis sp. PCC 6803. Sci Rep 8:10305

40. Touloupakis E, Cicchi B, Benavides AMS, Torzillo G (2016) Effect of high pH on growth of Synechocystis sp. PCC 6803 cultures and their contamination by golden algae (Poterioochromonas sp.). Appl Microbiol Biotechnol 100:1333-1341 
41. Schwarz R, Forchhammer K (2005) Acclimation of unicellular cyanobacteria to macronutrient deficiency: emergence of a complex network of cellular responses. Microbiology 151:2503-2514

42. Yao B, Xi B, Hu C, Huo S, Su J, Liu H (2011) A model and experimental study of phosphate uptake kinetics in algae: considering surface adsorption and P-stress. J Environ Sci 23:189-198

43. Zhang H-L, Fang W, Wang Y-P, Sheng G-P, Zeng RJ, Li W-W, Yu H-Q (2013) Phosphorus removal in an enhanced biological phosphorus removal process: roles of extracellular polymeric substances. Environ Sci Technol 47:11482-11489

44. Zhou Y, Nguyen BT, Zhou C, Straka L, Lai YS, Xia S, Rittmann BE (2017) The distribution of phosphorus and its transformations during batch growth of Synechocystis. Water Res 122:355-362

45. Zerulla K, Ludt K, Soppa J (2016) The ploidy level of Synechocystis sp. PCC 6803 is highly variable and is influenced by growth phase and by chemical and physical external parameters. Microbiology 162:730-739
46. Campos-Rudin M, Silva-Benavides AM (2018) Ammonium and phosphate removal using batch laboratory cultures by microalgae and cyanobacteria isolated from Costa Rica water bodies. Int J Trop Biol 66:S83-S91

47. Rojsitthisak P, Burut_Archanai S, Pothipongsa A, Powtomgsook $S$ (2017) Repeated phosphate removal from recirculating aquaculture system using cyanobacterial remediation and chitosan flocculation. Water Environ J 31:598-602

48. Kabariya JH, Ramani VM (2018) Dairy wastewater treatment by cyanobacteria for removal of nutrients with extraction of high value compounds from biomass. Int J Curr Microbiol App Sci $7: 1527-1538$

Publisher's Note Springer Nature remains neutral with regard to jurisdictional claims in published maps and institutional affiliations. 